\title{
Development and Uncertainty Evaluation of Calibrating System for Digital Energy Setting
}

\author{
Sha Zhao ${ }^{1, a}$, Jingfen Bai ${ }^{1}$, Fantao Lin ${ }^{1}$, Xiangjiang Yang ${ }^{1}$ and Chunping $\mathrm{Yu}^{1}$ \\ ${ }^{1}$ China Electrical Power Research Institute, 100192 Beijing, China
}

\begin{abstract}
The power measurement mode has made fundamental changes with the development of smart substation, traditional energy metering analog devices are replaced by digital devices according to the requirements of digitalized information substation. Creating traceability between digital measuring value and analog measuring value and developing relevant calibration devices are key issues in smart substation construction. A new device for calibration digital energy meters using integral calibration technology is described in this paper. Based on the Standard Comparison Method, the device establishes a connection between the digital power and the traditional analog power with the integral calibration technology, and solves the problem that digital power was not able to be traced. The device is designed with final measuring voltage level: $220 \mathrm{kV} / 110 \mathrm{kV}$, measuring current range: $5 \mathrm{~A} \sim 5000 \mathrm{~A}$, and extended uncertainty less than $0.05 \%$. Furthermore, the estimating process of the uncertainty of the device is discussed emphatically.
\end{abstract}

\section{Introduction}

Smart grid construction is an important field of growing concern in the world, and the smart substation is one of the important nodes in building smart grid, which bears electricity settlement gateway function and is an important guarantee for power efficient transmission and reliable operation of grid.

Different from traditional substation, the communication platform of smart substation is established on the basis of IEC61850 international unified network communication standard [1-2]. The electric energy metering device in smart substation contains Electronic Transformer, Merging Unit and Digital Input Electricity Meter, these components are fundamentally different from traditional ones just like traditional electromagnetic Transformer and Electricity Meter on the methods of measuring principle, and data transmission. Their metrological characteristics are clearly different from traditional energy metering device too. Analog signals are transmitted by cable in the traditional power measurement mode, while digital signals are transmitted by fibre optic Ethernet in the digital power measurement mode, so the traditional calibration method and device cannot meet the requirement of digital electric energy metering system.

In addition, metering is featured by scientific and legality, and is the process to unify the unit and make the measuring value accurate and reliable. The electric energy measurement device used for trade account must be traced to the national standard indirectly by compulsory verification. Up till

\footnotetext{
a Corresponding author : zhaosha@epri.sgcc.com.cn
} 
now, China does not yet possess the capability of digital electric energy metering traceability. Fortunately, many scholars have made lots of related researches on calibration methods and traceability, and have come to many important conclusions [3-7]. However, most of these researches consider Electronic Transformer and Merging Unit as a whole for calibrating, while the Digital Input Electricity Meters are calibrated separately, there are not many studies by considering all digital electric energy measurement device as a whole [8-10], and are still not very mature.

The tests of Electronic Instrument Transformer (ECT/EVT) are commonly carried out with the help of Merging Unit (MU), ECT/EVT and MU are indivisible in practice, such as the tests of clock synchronization and data modification, it is difficult to make separate calibration for them. As a power calculation and cumulative unit, the Digital Input Electricity Meter does not have any measuring function for physical parameters, so there should be no error in it [11]. The quantization error, synchronization error, even network traffic anomaly from Merging Unit will all have an impact on Digital Input Electricity Meter. In addition, as one of the smart substation equipments, there are many parameters to be set for the Digital Input Electricity Meter during the debugging process of smart substation, which will also affect the calibration.

Aiming at making the digital power be able to trace to the traditional power standard, this paper presents an integral calibration method by considering the digital electric energy measurement device as a whole, to avoid the problem that Electronic Transformer and Merging Unit can't be calibrated separately, to calibrate the composite error of the whole set more comprehensively, and to reflect the performance of the whole set, including but not limited to network status.

\section{Principle Introduction}

Based on the Standard Comparison Method, our digital electric energy calibration system can measure the global error of electric energy measurement device. It is a single phase digital input energy standard device, and its principle block diagram is as shown in Figure 1:

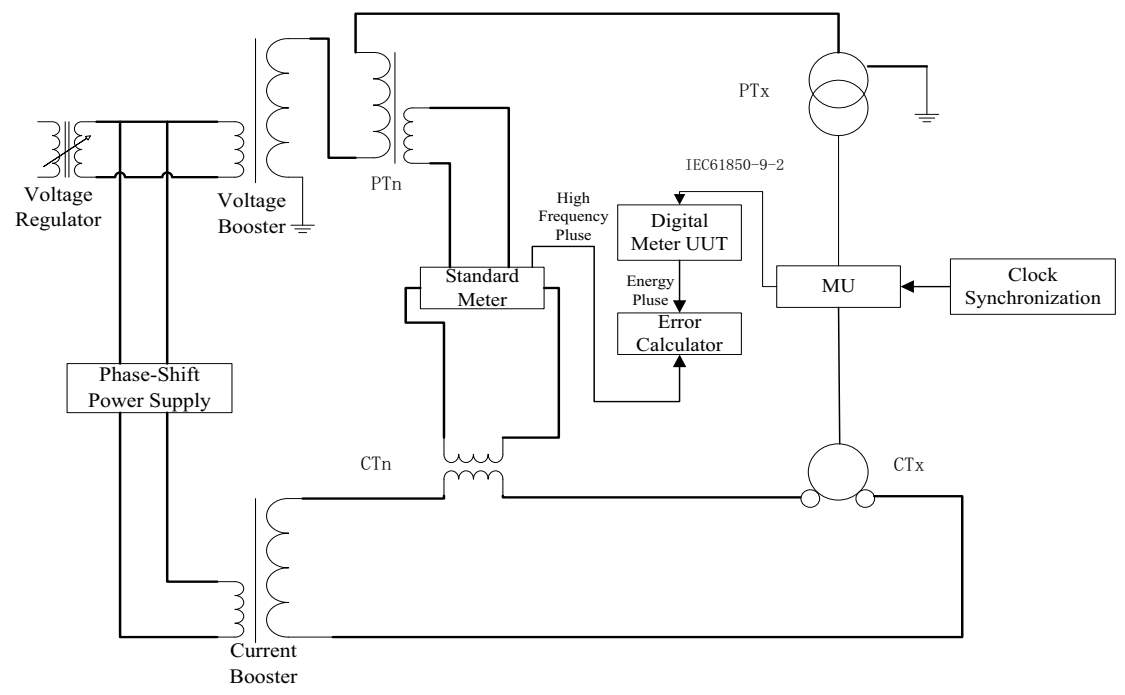

Figure 1. The principle block diagram of electric energy standard device

The phase-shift power supply shifts the voltage phase angle in voltage circuit and current circuit, and generates two different phases of $\mathrm{AC}$ voltages according to the phase of test point. Using a voltage regulator and a voltage booster, the input voltage in voltage circuit can be convert to high voltage and output to the Electronic Voltage Transformer PTx of the digital energy measurement device under test and the standard Voltage Transformer PTn of the standard equipment, the input voltage in current circuit through a voltage regulator and a current booster can be converted to high 
current and output to the Electronic Current Transformer CTx of the digital energy measurement device under test and the standard Current Transformer CTn.

The digital energy measurement device is made up by an Electronic Current Transformer CTx, an Electronic Voltage Transformer PTx, a Merging Unit and a Digital Input Electricity Meter under test. For digital output Electronic Transformer, ECT/EVT converts primary current (voltage) signal to digital signal and send to Merging Unit over optical fiber. For analog output Electronic Transformer, ECT/EVT converts primary current (voltage) signal to secondary small voltage signal and sends the signals to Merging Unit over shield cable. The A/D converter in Merging Unit transforms analog electronic signals into digital sampling values. These digital current and voltage sampling values can be assembled into network frames according to the IEC61850-9-2 standard by Merging Unit and sent to Digital Input Electricity Meter, which extracts current and voltage information from network frames to calculate electric energy, and sends out low-frequency electric energy pulses.

On the other hand, the electric energy standard device is made up by a traditional standard Current Transformer CTn, a standard Voltage Transformer PTn and a standard Electric Energy Meter. The traditional standard Energy Meter measures the standard electric energy directly using the output secondary signals from standard Current Transformer CTn and standard Voltage Transformer PTn, and then sends out high - frequency electric energy pulses. Low-frequency electric energy pulses from Digital Input Electricity Meter and high - frequency electric energy pulses from standard Energy Meter are putted into an error calculator simultaneously, so the global error of digital energy measurement device can be obtained.

According to the IEC61850-9-2 standard, the sampled values that the Digital Input Electricity Meter received are the primary current and voltage of the digital transformer, so the output pulse of the Digital Input Electricity Meter is proportional to the primary electric energy pulse. The following equation should apply to calculate the global error of the digital energy measurement device under test:

$$
\gamma=\frac{E_{\mathrm{X}}-K_{U} K_{I} E_{\mathrm{N}}}{K_{U} K_{I} E_{\mathrm{N}}} \times 100 \%
$$

where

$E_{\mathrm{N}}-$ energy value of analog standard Energy Meter tested

$E_{\mathrm{X}}$ - energy value of Digital Input Electricity Meter tested

$K_{U}$-transformation ratio of standard Voltage Transformer

$K_{\Gamma}$ transformation ratio of standard Current Transformer

The standard Voltage Transformer has four taps: $\frac{220 \mathrm{kV}}{100 \mathrm{~V}}, \frac{220 \mathrm{kV} / \sqrt{3}}{100 \mathrm{~V}}, \frac{110 \mathrm{kV}}{100 \mathrm{~V}}$ and $\frac{110 \mathrm{kV} / \sqrt{3}}{100 \mathrm{~V}}$. The standard Current Transformer is of wide-range with the primary rated current 5A 2000A, and the secondary rated current $5 \mathrm{~A}$. These transformers meet the requirements of energy measurement device calibration of the substation which has voltage levels of $110 \mathrm{kV}, 220 \mathrm{kV}$ and various loads. With a synchronous clock device which has optoelectronic pulse conversion function, high precision clock synchronization is realized between the standard device and the Digital Input Electricity Meter under test. In such a way, the additional error caused by synchronization is avoided.

\section{Uncertainty Analysis}

\subsection{Mathematical model}

Using direct comparison method, the measurement error $\gamma$ of the digital energy measurement device can be calculated by using the following formula: 


$$
\gamma=\frac{E_{\mathrm{X}}-K_{V} K_{I} E_{\mathrm{N}}}{K_{V} K_{I} E_{\mathrm{N}}} \times 100 \%
$$

where

$E_{\mathrm{N}}$ - energy value of analog standard Energy Meter tested

$E_{\mathrm{X}}$ - energy value of Digital Input Electricity Meter tested

$K_{U}$-transformation ratio of standard Voltage Transformer

$K_{I}$ transformation ratio of standard Current Transformer

\subsection{Sources of uncertainty}

According to the principle and the mathematical model of the device, it can be made out that the sources of uncertainty include the following parts:

(1) Uncertainty of the digital energy measurement device under test, mainly refers to the uncertainty component introduced by random factors such as stability.

(2) Uncertainty component introduced by the ratio error and phase error of Voltage Transformer.

(3) Uncertainty component introduced by the ratio error and phase error of Current Transformer.

(4) Uncertainty component introduced by the standard Energy Meter.

This paper only analyses the uncertainty components introduced by the standard device, do not contain the uncertainty component introduced by the digital energy measurement device to be tested. Based on the Standard Comparison Method, the standard device and the device under test are connected with the same set of voltage and current circuit, therefore, the contribution of power supply to the uncertainty of the whole device can be ignored.

\subsection{Uncertainty component introduced by ECT/EVT}

For convenience, we will analyze the uncertainty components step by step. First, transform section of the transformer is discussed. The influence of transformer on electric energy of output terminal is presented here.

Instead of making modifications on the ratio error and phase error traceable value of ECT/EVT, uncertainty was evaluated by the accuracy class of the transformer itself. Input voltage and current are putted into the standard Energy Meter through ECT/EVT, the influence on electric energy should include ratio error and phase error.

The ratio error $f_{V}\left(f_{I}\right)$ can be calculated by:

$$
\begin{aligned}
& f_{V}=\frac{K_{\mathrm{V}} V_{2}-V_{1}}{V_{1}} \times 100 \% \\
& f_{I}=\frac{K_{I} I_{2}-I_{1}}{I_{1}} \times 100 \%
\end{aligned}
$$

where

$$
\begin{aligned}
& V_{2}\left(I_{2}\right) \text { - the actual second voltage(current) } \\
& K_{V}\left(K_{I}\right) \text {-rated transformation ratio } \\
& V_{1}\left(I_{1}\right) \text { - the actual primary voltage (current) }
\end{aligned}
$$

Phase error ${ }_{\nu}\left(\delta_{I}\right)$ is also called angle error, which can be expressed as the phase displacement between the vectors that obtained by revolving the secondary voltage (current) vector $V_{2}\left(I_{2}\right)$ for 180 degrees counterclockwise and the primary voltage (current) vector $V_{I}\left(I_{I}\right)$.

According to the calculation formula of single-phase electric energy $P=V I \cos \varphi$, the following 
is valid:

$$
\begin{aligned}
& \frac{\partial P}{\partial V}=I \cos \varphi \\
& \frac{\partial P}{\partial I}=V \cos \varphi \\
& \frac{\partial P}{\partial \varphi}=-V I \sin \varphi
\end{aligned}
$$

$V, I$ and $\varphi$ are mutual independence, so according to the uncertainty conduction formula, $\Delta P$ can be calculated from:

$$
\begin{aligned}
\Delta P & =\sqrt{\left(\frac{\partial P}{\partial V} \Delta V\right)^{2}+\left(\frac{\partial P}{\partial I} \Delta I\right)^{2}+\left(\frac{\partial P}{\partial \varphi} \Delta \varphi\right)^{2}} \\
& =\sqrt{(I \cos \varphi \Delta V)^{2}+(V \cos \varphi \Delta I)^{2}+(V I \sin \varphi \Delta \varphi)^{2}}
\end{aligned}
$$

Divided by active power deviations:

$$
\frac{\Delta P}{P}=\frac{\sqrt{(I \cos \varphi \Delta V)^{2}+(V \cos \varphi \Delta I)^{2}+(\mathrm{VI} \sin \varphi \Delta \varphi)^{2}}}{V I \cos \varphi}
$$

Put the formula (3), formula (4) into formula (7):

$$
\begin{gathered}
=\sqrt{\left(\frac{\Delta V}{V}\right)^{2}+\left(\frac{\Delta I}{I}\right)^{2}+(\operatorname{tg} \varphi \cdot \Delta \varphi)^{2}} \\
=\sqrt{{f_{V}}^{2}+f_{I}^{2}+\operatorname{tg}^{2} \varphi \cdot \Delta \varphi^{2}}
\end{gathered}
$$

When $\Delta \varphi=\delta_{V}-\delta_{I}$, formula (8) is expressed as:

$$
=\sqrt{f_{\mathrm{V}}^{2}+f_{\mathrm{I}}^{2}+\operatorname{tg}^{2} \varphi \cdot \delta_{V}^{2}+\operatorname{tg}^{2} \varphi \cdot \delta_{I}^{2}-2 \operatorname{tg}^{2} \varphi \cdot \delta_{V} \delta_{I}}
$$

When the variation of $V, I, \varphi$ is very small, the unrelated higher order term could be neglected, meanwhile, $\delta_{V} \approx \sin \delta_{V}, \delta_{I} \approx \sin \delta_{I}$, then,

$$
\frac{\Delta P}{P}=\sqrt{f_{V}^{2}+f_{\mathrm{I}}^{2}+\operatorname{tg}^{2} \varphi \cdot \sin \delta_{V}^{2}+\operatorname{tg}^{2} \varphi \cdot \sin \delta_{V}^{2}}
$$

where

$$
\begin{aligned}
& f_{V}=f_{I}=1 \times 10^{-4} \\
& \delta_{V}=\delta_{I}=0.3 \\
& \sin \delta_{V}=\sin \delta_{V}=87.3 \times 10^{-6}, k=\sqrt{3}
\end{aligned}
$$

\section{4 uncertainty component introduced by standard energy meter}

This device does not use the tracing error to correct the measurement results, but only through tracing to confirm the error limit of accuracy class that the standard Energy Meter met. Therefore, the contribution of the standard Energy Meter to the digital energy standard device is equal to the 
accuracy class $0.01 \%$, which is processed by the average distribution, $\mathrm{k}=\sqrt{3}$, the standard uncertainty of this component can be given as:

$$
u_{\mathrm{rel}}\left(E_{\mathrm{N}}\right)=0.01 \% / \sqrt{3}=57.7 \times 10^{-6}
$$

\subsection{Uncertainty component introduced by clock synchronization}

The uncertainty introduced by clock synchronization device will have an effect on the clock of the Merging Unit and the measurement uncertainty of the whole set. The synchronization accuracy of the clock synchronization device is less than 100ns. By processing it with the average distribution, the relative standard uncertainty $\mathrm{u}_{2}$ can be given as:

$$
u_{2}=\frac{100 \mathrm{~ns}}{1 \mathrm{~s}} \cdot \frac{1}{\sqrt{3}}=5.8 \times 10^{-8}
$$

\subsection{Combined standard uncertainty}

The proportional change part of the voltage (current) and the electric energy measurement part of the standard Energy Meter are independent of each other, so the combined standard uncertainty of the whole set can be given as:

$$
u_{1}=\frac{0.001 \%}{2 \sqrt{3}}=2.9 \times 10^{-6}
$$

\begin{tabular}{|c|c|c|c|c|c|c|c|c|c|}
\hline \multirow{2}{*}{ NO } & \multirow{2}{*}{\multicolumn{2}{|c|}{ Uncertainty Factors }} & \multirow{2}{*}{$\begin{array}{l}\text { Sub-item } \\
\text { Uncertaint } \\
\mathrm{y}(\times 10-6)\end{array}$} & \multirow{2}{*}{$\begin{array}{l}\text { Coverage } \\
\text { Factor }\end{array}$} & \multirow{2}{*}{$\begin{array}{c}\text { Standard } \\
\text { Uncertainty } \\
\text { Component } \\
\quad\left(\times \mathbf{1 0}^{-6}\right) \\
u_{i}(x)\end{array}$} & \multirow{2}{*}{$\begin{array}{l}\text { Propagation } \\
\text { Coefficient }\end{array}$} & \multicolumn{3}{|c|}{$\begin{array}{c}\text { Uncertainty }\left(\times 10^{-6}\right) \\
u_{i}(y)\end{array}$} \\
\hline & & & & & & & $\begin{array}{c}\mathrm{PF}= \\
1.0\end{array}$ & $\begin{array}{c}\mathrm{PF}= \\
0.5\end{array}$ & $\begin{array}{c}\mathrm{PF}= \\
0.8\end{array}$ \\
\hline 1 & \begin{tabular}{|l|} 
Uncertainty \\
Component \\
Introduced B \\
Standard Ene \\
Meter \\
\end{tabular} & & 100 & $\sqrt{3}$ & 57.7 & 1 & 57.7 & 57.7 & 57.7 \\
\hline \multirow{4}{*}{2} & \multirow{4}{*}{\begin{tabular}{|l|} 
Uncertainty \\
Component \\
Introduced \\
By Standard \\
Transformer \\
\end{tabular}} & $f_{V}$ & 100 & $\sqrt{3}$ & 57.7 & 1 & 57.7 & 57.7 & 57.7 \\
\hline & & $f_{I}$ & 100 & $\sqrt{3}$ & 57.7 & 1 & 57.7 & 57.7 & 57.7 \\
\hline & & $\delta_{V}$ & 87.3 & $\sqrt{3}$ & 50.4 & $\operatorname{tg} \varphi$ & 0 & 87.3 & 40.4 \\
\hline & & $\delta_{I}$ & 87.3 & $\sqrt{3}$ & 50.4 & $\operatorname{tg} \varphi$ & 0 & 87.3 & 40.4 \\
\hline 3 & \multicolumn{2}{|c|}{$\begin{array}{l}\text { Uncertainty } \\
\text { Component Introduced } \\
\text { By Error Processor }\end{array}$} & 10 & $2 \sqrt{3}$ & 2.9 & 1 & 2.9 & 2.9 & 2.9 \\
\hline 4 & \multicolumn{2}{|c|}{$\begin{array}{l}\text { Uncertainty } \\
\text { Component Introduced } \\
\text { By Synchronous } \\
\text { Device }\end{array}$} & 0.1 & $\sqrt{3}$ & 0.06 & 1 & 0.06 & 0.06 & 0.06 \\
\hline \multicolumn{7}{|c|}{ Combined Standard Uncertainty } & 100 & 159 & 116 \\
\hline
\end{tabular}

Table 1. Uncertainty summary table.

\subsection{Expanded uncertainty}

Expanded uncertainty $U_{\text {rel }}(P)=k u_{\text {rel }}(P), k=2$, then the expanded uncertainty of the digital energy standard device can be expressed as:

$$
U_{\text {rel }}(P)=2 \sqrt{f_{V}^{2}+f_{I}^{2}+\operatorname{tg}^{2} \varphi \cdot \sin \delta_{V}^{2}+\operatorname{tg}^{2} \varphi \cdot \sin \delta_{V}^{2}+u_{\text {rel }}^{2}\left(E_{N}\right)+u_{1}^{2}+u_{2}^{2}}
$$

Expanded uncertainty is as shown in the following table: 
Table 2. Expanded uncertainty

\begin{tabular}{|c|c|}
\hline Power Factor & Expanded Uncertainty $(\%)$ \\
\hline $\cos \varphi=1.0$ & 0.020 \\
\hline $\cos \varphi=0.5 \mathrm{~L}(0.5 \mathrm{C})$ & 0.032 \\
\hline $\cos \varphi=0.8 \mathrm{C}(0.8 \mathrm{~L})$ & 0.024 \\
\hline
\end{tabular}

Considering the influence of the random error caused by noise and measurement stability, the overall measurement uncertainty is less than $0.05 \%$.

\section{Conclusion}

Along with the expansion of smart substation construction, digital electric energy device has been widely applied, in order to ensure the accuracy of electric energy metering, it must trace to the electric energy metering standard of higher level. In this paper, a new digital energy standard device is designed, where integral calibration technology is used to complete the tracing of electric energy. The expanded uncertainty of the device can reach $0.05 \%(k=2)$, that can meet the requirements of the calibration and acceptance tests of all levels of digital electric energy measurement devices with voltage level: $110 \mathrm{kV} / 220 \mathrm{kV}$, current range: $5 \mathrm{~A} \sim 5000 \mathrm{~A}$, and integrated error less than $0.1 \%$.

By taking the traditional electric energy metering system as standard, a connection between digital power and traditional analog power is established. And by applying integral calibration method, the additional error caused by tracing digital power to traditional analog power when make separate calibration for digital input electricity meter is avoided, the unified and accurate measuring value is effectively guaranteed. The device provides a simple and effective way to make the digital electric energy metering technology meet the requirements of law, and provides reference for China to establish a national digital energy metering traceability system.

\section{Acknowledgement}

Supported by the National High Technology Research and Development of China("863" Project)(Grant No. 2015AA050404)

\section{References}

1. Y.M. Ren, L.J. Qin, Q.X. Yang, Automat. Electr. Pow. Syst 24, 8(2000)

2. W.S. Tan, Power. Syst. Technol 25,9(2001)

3. L. Yao, S.T. Li, C.G. Lu, Electr. Meas. Instr 47, 28-32(2010).

4. G.Y. Lin, S.L. Zhou, W.M. Sun, S.C. Wu, Proc. CSU-EPSA 23, 3(2011).

5. Y. Xiao,B. Jiang,W. Zhao,B. Ai, S.L. Huang, Y. Tang, X.S. Chen, Electr. Meas. Instr 51,1(2014)

6. R.M. Chen, X. Li, Z.M. Kong, Y.J. Fang, Electr. Meas. Instr 49, 10(2012)

7. R.M. Chen, Z.Y. Wang, Z.M. Kong, Y.J. Fang, Electr. Meas. Instr 49, 9(2012)

8. Z. Xu, S. Zhao, R.M. Chen, Y. Xiao, W.M. Sun, Control. Instrum. Chem. Ind 12(2012)

9. W.M. Sun, G.Y. Lin, S.L. Zhou, K.C. Li, Electr. Meas. Instr 47, 8(2010)

10. Q. Li, S.H. Zhang, Y.B. Lu, D. Han, H.L. Hu, H. Li, D.Y. Li, Electr. Meas. Instr 47, 10(2010)

11. H.J. Yu, YunNan. Electr. Pow 36, 5(2008) 